\title{
Entwurf einer universellen tastenlosen optischen Fernbedienung
}

\author{
Heinrich Ruser \\ Lehrstuhl für Angewandte Physik und Messtechnik, Universität der Bundeswehr München, Neubiberg \\ Kontakt: heinrich.ruser@unibw.de
}

\section{Einleitung}

Im Beitrag wird der Entwurf eines optischen Systems zur universellen Fernbedienung vieler technischer Geräte und Systeme im Haushalt, in Gebäuden oder in IndustrieUmgebungen beschrieben: Mittels eines leichten und tastenlosen "Lichtzeigers", der wie eine kleine Taschenlampe in der Hand liegt, werden quasi-intuitive Zeige- und Bewegungsgesten in Richtung des zu bedienenden Gerätes ausgeführt, um dieses auszuwählen, zu schalten oder dessen Einstellungen in Stufen oder stufenlos zu verändern. Diese natürliche Mensch-Maschine-Schnittstelle soll insbesondere dazu beitragen, Personen mit eingeschränkter Mobilität und älteren Menschen ein längeres selbständiges Leben in den eigenen "vier Wänden“ zu erleichtern. Aber auch für andere Nutzergruppen dürfte die einfache Bedienbarkeit und die universelle Einsetzbarkeit des "Lichtzeigers" ein Zugewinn an Komfort und Bequemlichkeit bedeuten. Der Verzicht auf zu erlernende Gesten und der ausgesprochen geringe Hardware-Aufwand stellen dabei wesentliche Unterscheidungen zu alternativen Ansätzen dar.

\section{Motivation}

Zeige- und Bewegungsgesten sind uns sehr vertraut, wir empfinden sie als natürlich und setzen sie in vielen Alltagssituationen quasi-intuitiv ein. Die Bedienung technischer Geräte im Wohn- und Arbeitsumfeld geschieht jedoch weiterhin überwiegend mit Knöpfen und Schaltern oder mittels verschiedener gerätespezifischer, tastenbasierter Infrarot-Fernbedienungen. Im Beitrag wird eine intuitive gestenbasierte Fernbedienung vorgestellt, die die Gerätebedienung vereinfachen, vereinheitlichen und insgesamt komfortabler machen soll. Insbesondere für Personen mit eingeschränkter Mobilität und ältere Menschen ist dies von hoher Bedeutung, um länger ein selbstbestimmtes Leben im eigenen Wohnumfeld führen zu können.

Die Vision einer solchen gestenbasierten Fernbedienung war in Grundzügen bereits 2008 veröffentlicht worden [1]. In der Zwischenzeit sind vielfältige Lösungsansätze für die gestenbasierte Mensch-Maschine-Interaktion bekannt geworden. In ausgewählten Anwendungsbereichen wurden diese bereits kommerzialisiert (u.a. stifffreie Dokumentation, virtuelle Montage, häufig in Verbindung mit Augmented bzw. Virtual Reality-Datenbrillen) oder sind sogar im Massenmarkt angekommen (Gestensteuerung auf dem Smartphone, im Automobil) [2-4]. Die Entwicklung konzentrierte sich dabei auf die Nahdistanz-Erfassung von Freihand-(Finger-) Gesten über einem Display, mehrheit- lich mit 2D- oder 3D-Kameras [5] oder ortssensitiven kapazitiven Sensor-Arrays [6].

Geräte in einer Entfernung von mehreren Metern oder große interaktive Display-Wände werden intuitiv durch mit Armbewegungen "in die Luft gezeichnete“ Gesten "gesteuert". Die Erfassung dieser räumlichen FreihandGesten („mid-air gestures“) [7,8] erfordert jedoch einen ungleich höheren Hardware- und Software-Einsatz als die Erfassung aus Nahdistanz.

Abhilfe schafft ein physisches Gerät, das in der Hand gehalten oder am Arm getragen wird (,tangible $\mathrm{HCl}$ ). Neben Datenhandschuhen und Armbändern [9] werden dazu heute vielfach Smartphones und andere mobile Endgeräte empfohlen, die mit entsprechenden Steuereinheiten beispielsweise im „Smart Home“ gekoppelt sind und mit denen auf der Touchscreen-Oberfläche oder mittels Gesten, erfasst z.B. durch die Beschleunigungssensoren im Smartphone, ausgewählte Geräte gesteuert werden können (siehe u.a. [10]).

Für viele, vor allem ältere Menschen, ist dies jedoch keine erstrebenswerte Option: Abgesehen davon, dass vielfach die Hausgeräte-Bedienung via Smartphone als unnatürlich und umständlich empfunden wird, ist der Einsatz des teuren Smartphones von der steten Sorge begleitet, dass es herunterfallen und beschädigt werden könnte.

Neue Entwicklungen in diesem Bereich sollten einfache und einfach nachvollziehbare Bedienungen ermöglichen, die eine natürliche, reibungslose Mensch-TechnikInteraktion gewährleisten. Aus dieser Grundüberlegung resultiert der im Folgenden vorgestellte Lösungsansatz.

\section{Grundaufbau des Systems}

Die gestenbasierte tastenlose optische Fernbedienung setzt sich aus folgenden Grundelementen zusammen (siehe Abb. 1):

1. ein batteriebetriebenes, tastenloses Handgerät („SmartPointer") ähnlich einer kleinen Taschenlampe, das zum Auswählen eines bestimmten Gerätes sichtbares Licht und für die Gerätebedienung strukturiertes Infrarotlicht aussendet,

2. ein oder mehrere Arrays von lichtempfindlichen Empfängern, welche an den oder in der Nähe der zu bedienenden Geräte angebracht werden, sowie

3. eine Dekodier- und Kommunikationseinheit zur Rekonstruktion und Erkennung der ausgeführten Handbewegungen sowie deren Umsetzung in die jeweiligen gerätespezifischen Steuerbefehle und Anbindung an das zu bedienende Gerät. 


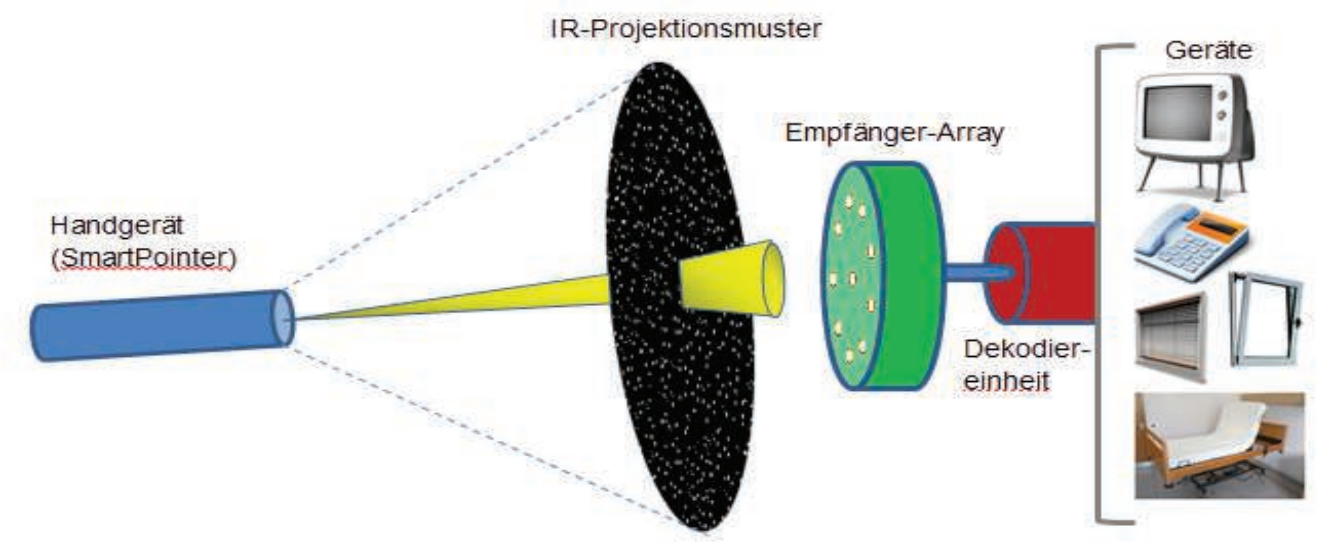

Abb. 1: Schematischer Aufbau der optischen Fernbedienung, bestehend aus 1) Handgerät mit ausgesendetem sichtbaren Lichtstrahl (gelb) sowie Infrarot-(IR-) Projektionsmuster, 2) Empfänger-Array an oder in der Nähe des zu bedienenden Gerätes sowie 3) gerätespezifischer Dekodiereinheit der erkannten Gesten (von links nach rechts).

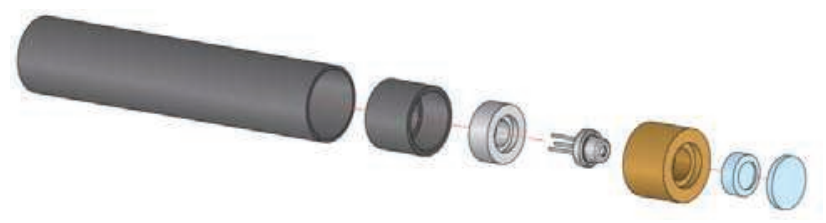

Abb. 2: Schematischer Aufbau des stiftähnlichen Handgerätes, mit Laserdiode, Kollimator-Linse und Diffraktivem Optischem Element (DOE). Den größten Raum nimmt die Batterie ein. (C) LaserComponents)

Die Gerätebedienung mit dem stiftähnlichen Handgerät erfolgt über intuitive Zeige- und Bewegungsgesten (wobei die Lichtaustrittsseite in Richtung des zu bedienenden $\mathrm{Ge}-$ räts zeigt) ohne vorheriges Erlernen von Regeln und knüpft stattdessen an das Erfahrungswissen über Gesten im Alltag an:

- Die Auswahl eines bestimmten Gerätes erfolgt durch längeres Zeigen mit dem sichtbaren Lichtstrahl auf das gewünschte Gerät (Lichtschalter, Radio, Fernseher, Jalousie, Heizung etc.). Durch die Öffnungsweite des sichtbaren Strahlkegels ist gewährleistet, dass der gerätenahe Lichtempfänger diese Information aufnimmt.

- Die Bedienung erfolgt durch Bewegung des Handgerätes mittels typischer Gesten. Dadurch wird das im Handgerät erzeugte Infrarot-Projektionsmuster relativ zum feststehenden Empfängerarray am Gerät bewegt. Aus der Korrelation der Intensitätsverläufe an verschiedenen Fotodioden-Paaren in bekannter räumlicher Anordnung zueinander werden die Geschwindigkeitsvektoren des Musters im Zeitverlauf berechnet und daraus die GestenTrajektorien rekonstruiert. Diese Trajektorien werden dann im Ergebnis einer Multi-Klassen-Klassifikation vorher vereinbarten oder eingelernten Gesten zugeordnet, in entsprechende gerätespezifische Bedienfunktionen umgesetzt und an das Gerät übertragen.

Das Projektionsmuster des IR-Strahlenkegel (siehe Abb. 1) hat am Auftreffort in typischen Entfernungen von 1 bis ca. $6 \mathrm{~m}$ vom Handgerät einen großen Durchmesser von wenigstens $100 \mathrm{~cm}$, sodass auch "raumgreifende“ Gesten mit weiter Armausholung sicher detektiert werden.

Der Aufbau des Handgerätes ist schematisch in Abb. 2 gezeigt. Das batterieversorgte Handgerät enthält nur wenige, kommerziell verfügbare massentaugliche Komponenten. Dadurch kann es für jeden Nutzer mehrfach vorhanden sein. Das Handgerät kann in der Hemd- oder Hosentasche oder auch als Stift am Hals getragen werden. Um Energie zu sparen, aktivieren sich die Lichtquellen des Handgerätes nur bei Bewegung und bei einem Winkel zur Senkrechten, der größer ist als ein vorgegebener Grenzwert. Dies wird beispielsweise mittels eines MiniaturBeschleunigungssensors im Handgerät festgestellt.

\section{Projektionsmuster}

Das vom Handgerät ausgesendete räumlich strukturierte Licht wird durch ein speziell gestaltetes Dia vor der IRSendediode erzeugt. Die entstehende Intensitätsverteilung auf der Projektionsfläche (d.h. in der Empfängerebene) muss geeignet sein, Bewegungen dieser Abbildung durch Messung der Lichtintensität an den einzelnen Empfängerdioden und Anwendung von einfachen digitalen Signalverarbeitungsmethoden zu bestimmen.

Das Projektionsmuster ist so zu entwerfen, dass solche Intensitätsverteilungen in der Projektionsebene entstehen, dass für alle Bewegungen des Musters aus den kontinuierlich aufgenommenen Lichtintensitäten an den einzelnen Empfängerdioden eine eindeutige Berechnung des zweidimensionalen Positions-, Geschwindigkeits- und Beschleunigungsvektors als Funktion der Zeit möglich wird. Diese Bedingung ist durch ein statistisch verteiltes Grauwert-Muster mit entsprechendem Raumfrequenzgehalt erfüllt. In Vorarbeiten sind für spezifische EmpfängerAnordnungen Grenzfrequenzen von Grauwert-Mustern für hohe Rekonstruktionsgüten bestimmt worden [11].

Zur Sicherstellung einer Reichweite von mehreren Metern verwendet das hier beschriebene System aus Kostenund Aufwandsgründen eine NIR-Laserdiode (Wellenlänge $905 \mathrm{~nm}$ ) in Kombination mit einer Kollimator-Linse und einem Diffraktiven Optischen Element (DOE). Mit dem DOE 
wird aus dem geeignet aufgeweiteten Laserstrahl mittels einer als Beugungsgitter wirkenden mikrostrukturierten Oberfläche eine Punktmatrix von bis zu mehreren Tausend Punkten erzeugt $[12,13]$. Neben der festzulegenden Laser-Wellenlänge sind die Größe und die statistische Anordnung der Beugungspunkte wesentliche Designparameter des DOE. In Abb. 3 sind ein Ausschnitt eines Punktmusters mit Pseudozufalls-Verteilung sowie eine Infrarot-Aufnahme mit erkennbarer Wiederholung des Musters dargestellt. In einem ersten Untersuchungsschritt werden solche Punktmuster in Simulationen erzeugt, mit den Parametern Punktgröße (,SpotSize'), mittlerer Punktabstand (,SpotDistance') und Punktdichte (,SpotDensity').
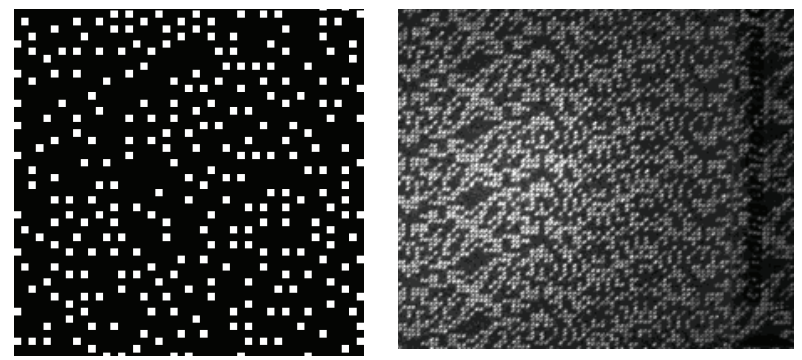

Abb. 3: DOE mit Pseudozufalls-Verteilung: Ausschnitt des Punktmusters aus Datenblatt und Infrarot-Aufnahme (C) LaserComponents)

\section{Empfängerarray und Rekonstruktion}

Ein weiterer Untersuchungsgegenstand ist die geeignete Anzahl und Anordnung der IR-lichtempfindlichen Empfänger (Fotodioden). Die bei Gestenausführung an den einzelnen Fotodioden aufgenommenen Lichtintensitäten werden simulationstechnisch nachgebildet, indem das Projektionsmuster entlang der Gesten-Trajektorien "bewegt“ wird. Aus den zeitlichen Intensitätsverläufen und der Größe der aktiven Diodenfläche werden die an den einzelnen Fotosensoren aufgenommenen elektrischen Signalverläufe bestimmt. Aus dem Vergleich von zeitlich aufeinander folgenden Aufnahmen der Intensitätsverteilung wird die Positionsänderung des Projektionsmusters mithilfe eines Kreuzkorrelationsverfahrens berechnet. Mittels der so bestimmten Zeitverschiebungen und der bekannten Empfängerpositionen werden die Geschwindigkeitsvektoren und die $x(t)$ - und $y(t)$-Koordinaten der Positionen der Projektionsmusters bestimmt und daraus die Trajektorie im Zeitverlauf zusammengesetzt (,rekonstruiert“) [11].

Die zur Berechnung der Geschwindigkeitsvektoren optimale geometrische Anordnung und Anzahl der Fotodioden ist abhängig vom Projektionsmuster und der Signalverarbeitung. Als Ergebnis der Untersuchungen mit verschiedenen Anordnungsformen wurde schließlich die radiale Anordnung in Verbindung mit Pseudo-Zufallsmustern wie in Abb. 3 dargestellt gewählt, siehe beispielhaft Abb. 4 . Die Optimierung erfolgt in einem mehrdimensionalen $\mathrm{Pa}-$ rameterraum (Projektionsmuster, Empfänger-Array, Signalverarbeitung). Die Simulationsergebnisse zeigten u.a., dass die Rekonstruktion mit steigender Anzahl der Foto- sensoren verbessert wird, wobei mit Hinblick auf die Komplexität des aufzubauenden Empfängers die Zahl der Fotosensoren (und notwendigen Empfangsverstärker) zunächst auf maximal 10 begrenzt wurde. Der Zuwachs an Rekonstruktionsqualität ist danach für die betrachtete Empfängeranordnung nur noch moderat.
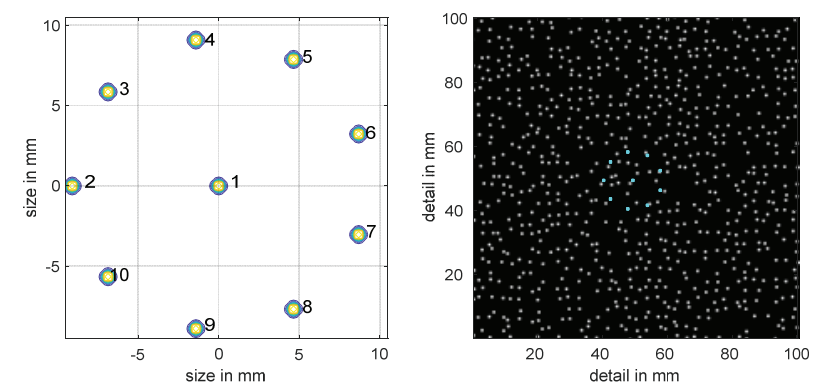

Abb. 4: Beispielhafte Anordnung der EmpfängerDioden (links) und hinterlegtes simuliertes Punktmuster mit Pseudozufalls-Verteilung (Ausschnitt) im gleichen Maßstab (rechts).

\section{Intuitives Gesten-Vokabular}

Für eine Vielzahl typischer Haushaltsgeräte sind in [1] Vorschläge zur gestenbasierten Ausführung grundlegender Bedienvorgänge mit dem SmartPointer gemacht worden. Lineare Bewegungen werden natürlicherweise eingesetzt, wenn etwas kontinuierlich verändert werden soll (Lautstärke, Helligkeit, Kippwinkel des Fensters), während mit ruckartigen Bewegungen oftmals stufenweise Änderungen wie eine Kanal- oder Titelwahl (in einem Abspielgerät) oder die Lüfter- und Gebläsestufe intendiert werden. Das System sollte also in der Lage sein, lineare Bewegungen (nach oben/unten/links/rechts) von ruckartigen Bewegungen in ebendiese Richtungen zu unterscheiden und damit verschiedenen Bedeutungen der Gerätebedienung zuzuordnen. Mit Drehbewegungen werden Veränderungen der Winkelstellung eines Drehreglers angezeigt (lauter/leiser, heller/dunkler, Lamellenstellungen der Jalousie oder Kippwinkel eines elektrisch zu öffnenden Fensters). Zahlen (z.B. zur direkten Kanalwahl) werden durch „in die Luft malen“ in Richtung der Empfänger übermittelt.

Für die aktuellen Untersuchungen wurden mit einer Gruppe von zunächst 20 Testpersonen einfache Bedienund Zeigegesten für bestimmte Gerätefunktionen erfasst [14]. Aufgrund der laufenden Entwicklung des SmartPointer-Systems erfolgte die Gestenausführung ersatzweise mit einem Laserpointer auf einer Projektionsfläche von ca. $1,5 \mathrm{~m} \times 1,5 \mathrm{~m}$ in einer Entfernung von ca. $2 \mathrm{~m}$ und die Aufzeichnung mit einer Videokamera. Die jeweiligen Gesten-Trajektorien wurden aus den zeitlich veränderlichen Positionen des Laserpunkt-Schwerpunktes abgeleitet.

Von vorerst 14 Probanden (74.8 \pm 3.3 Jahre) ohne erkennbare Tremor-Beeinträchtigung wurden mit dem Laserpointer jeweils mehrfach nacheinander typische quasiintuitive Bewegungen in 6 Klassen (Bewegung nach links/rechts, nach oben/unten, Kreisbewegung 
links/rechts) ausgeführt und als Referenztrajektorien (insgesamt mehr als 400) aufgezeichnet. In Abb. 5 sind für zwei Gestenklassen (Bewegung nach links sowie Kreisbewegung nach rechts) mehrere Gestenaufzeichnungen nach Vorverarbeitung (Zentrierung und Größennormierung) gezeigt.
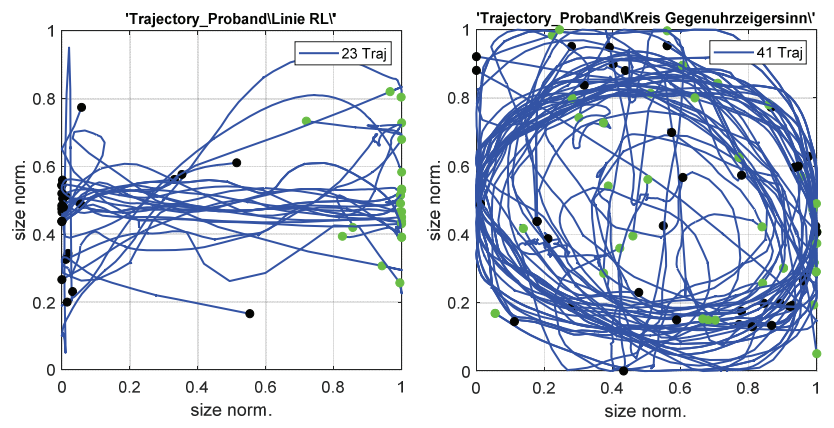

Abb. 5: Beispielhafte Referenztrajektorien für zwei Gestenklassen (Bewegung nach links sowie Kreisbewegung nach rechts). Die grünen Punkte kennzeichnen jeweils den Anfang der Gestenausführung, die schwarzen Punkte das Ende.

\section{Ergebnisse der Gesten-Rekonstruktion}

In Abb. 6 sind beispielhaft mit dem beschriebenen Verfahren der schrittweisen Gesten-Rekonstruktion ermittelte Bewegungen des Projektionsmusters entlang vorgegebenen Trajektorien dargestellt. Als Maß für die Güte der Rekonstruktion wird das auf die Gestenlänge bezogene geometrische Abstandsquadrat SSD (sum of squared differences) zwischen der vorgegebenen und der rekonstruierten Trajektorien gebildet.
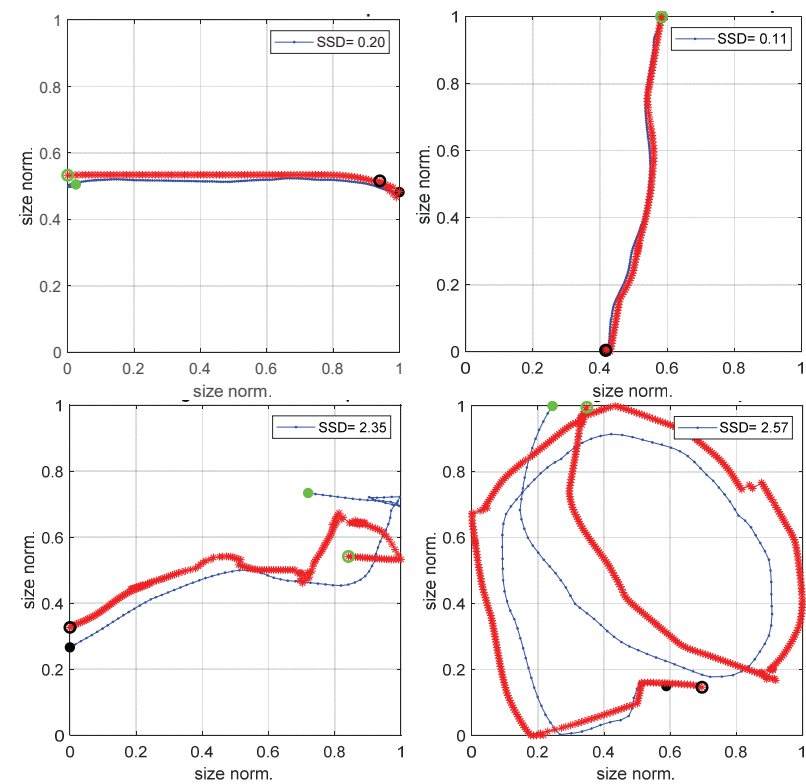

Abb. 6: Beispiele von Gesten-Rekonstruktionen (rote Linien) für zugrundegelegte Trajektorien (blau) mit Angabe des SSD-Wertes als Gütemaß der Rekonstruktion.
Für ausgewählte Fotodioden-Anordnungen ist das Projektionsmuster so zu gestalten, dass die Rekonstruktionsgüte hoch, d.h. das Abstandsmaß (SSD) der berechneten zur Referenztrajektorie so klein wie möglich ist.

Umfangreiche Simulationsrechnungen mit jeweils 100 verschiedenen Probanden-Trajektorien ergaben für optimierte Projektionsmuster und Empfänger-Anordnungen hohe mittlere Rekonstruktionsgüten (kleine SSD-Werte, idealerweise Null), siehe Abb. 7. Die Ergebnisse zeigen, dass insbesondere größere Punktgrößen (,SpotSize') und demgegenüber kleinere Diodenabstände für eine gute Gesten-Rekonstruktion zu bevorzugen sind, während die Abhängigkeit vom Punktabstand (,SpotDistance') weniger ausgeprägt ist. Dies hat praktische Bedeutung, denn mit größer werdendem Abstand des Musters von der Lichtquelle nimmt die Punktgröße zu, während der Punktabstand für eine Beugungsordnung festliegt. Projektionsmuster mit Punktgrößen $>5 \mathrm{~mm}$ sind nicht praxisrelevant und werden daher nicht betrachtet.
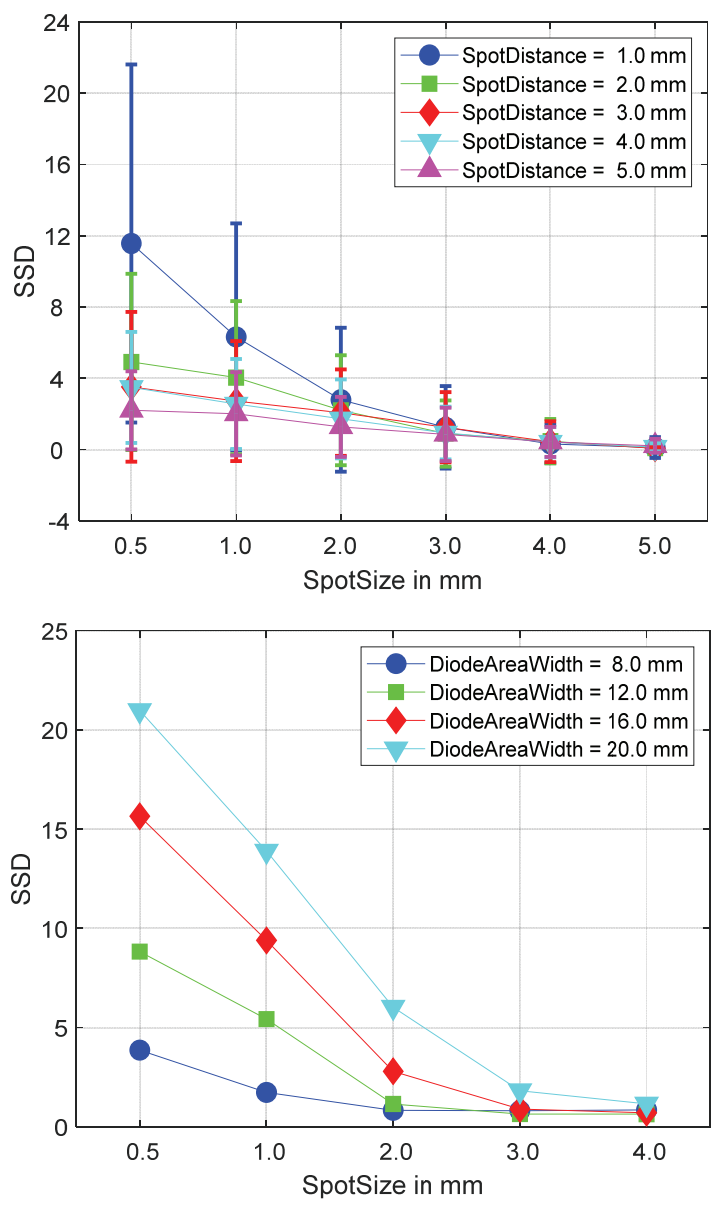

Abb. 7: Rekonstruktionsergebnisse (gemittelt über jeweils 100 verschiedene Trajektorien aus der GestenDatenbank) für Projektionsmuster mit unterschiedlichen Punktgrößen und mittleren Abständen zwischen zwei Punkten (oben) und für Punktmuster mit konstantem Punktabstand, aber veränderlicher Größe des EmpfängerArrays (,DiodeAreaWidth') und damit Abstand zwischen den Fotodioden (unten). 


\section{Diskussion und Ausblick}

Universal-Fernbedienungen für elektrische Geräte verfügen heute über unübersichtliche Bedienfelder, sind zu groß, zu schwer und nicht immer einfach bedienbar. Die angestrebte Entwicklung zielt auf die universelle Fernbedienung eines großen und erweiterbaren Kreises technischer Geräte und Systeme im Haushalt, in Gebäuden und in Industrieumgebungen durch einen universellen, kleinen, leichten, am Körper zu tragenden, preisgünstigen tastenlosen Lichtzeiger („SmartPointer“), dessen Einsatz quasiintuitiv ist: Die Auswahl/Aktivierung eines aus der Entfernung zu bedienenden Gerätes erfolgt durch kurzes Verweilen eines sichtbaren Lichtstrahles des SmartPointer auf dem Gerät. Die Fern-Bedienung geschieht mittels typischer, intuitiv im Bewusstsein verankerter Zeige- und Bewegungsgesten mit dem Lichtzeiger in Richtung auf das zu bedienende Gerät. Dadurch wird das ausgesendete Infrarot-Projektionsmuster mit pseudo-zufälliger Punktverteilung in seiner Intensität räumlich und zeitlich verändert. Aus den an feststehenden Fotodioden in der Nähe der Geräte empfangenen Intensitätsverläufen erfolgen mit geeigneten Signalverarbeitungsmethoden Rekonstruktion, Klassifikation und Interpretation der Gesten.

Umfangreiche Simulationsrechnungen zeigen für optimierte Projektionsmuster und verschiedene EmpfängerAnordnungen hohe mittlere Rekonstruktionsgüten (gekennzeichnet durch das normierte geometrische Abstandsmaß SSD zwischen vorgegebener und rekonstruierter Trajektorie). Aktuell werden experimentelle Untersuchungen mit kommerziellen Pseudo-Zufalls-DOEs vorbereitet. Weitere Schwerpunkte sind die automatische Trennung der aktiven, in den Gesten enthaltenen Bewegungen von den passiven Zu- und Rückführbewegungen über das charakteristische Geschwindigkeits- und Beschleunigungs-Zeit-Verhalten sowie der Einsatz adaptiver LernAlgorithmen zur Gestenklassifikation (siehe z.B. [15]).

\section{Literatur}

[1] RUSER, H., RUDLOFF, A., HORN, M.: Intuitive, gestenbasierte Fernbedienung mit einem ,SmartPointer' als Vision zur Optimierung der Mensch-MaschineSchnittstellen im Haushalt. In: Tagungsband 2. VDEFachkongress „Ambient Assisted Living”, Berlin, 2008.

[2] FEND, L, HOFMANN, J. (Hrsg.): Digitalisierung in Industrie-, Handels- und Dienstleistungsunternehmen Konzepte - Lösungen - Beispiele, Springer 2018.

[3] PREIM, B., DACHSELT, R.: Interaktive Systeme, Band 2: User Interface Engineering, 3D-Interaktion, Natural User Interfaces, Springer, Wiesbaden 2015.

[4] JAHANI, H., ALYAMANI, H., KAVAKLI, M, DEY, A., BILLINGHURST, M.: User evaluation of hand gestures for designing an intelligent in-vehicle interface, Lecture Notes in Computer Science (LNCS), vol.10243 (2017), S. 104-121.

[5] CHIU, P., KIM, Ch., ODA, H.: Recognizing gestures on Projected Button Widgets with an RGB-D camera, Proc. of the ACM Int. Conf. on Interactive Surfaces and Spaces (ISS'18), Tokyo 2018
[6] HASLINGER, L., HEHENBERGER, S., ZAGAR, B.: Capacitive Measurement System for Touchless Interaction, Proc. XXX. Eurosensors Conf., Budapest 2016.

[7] KOUTSABASIS, P., VOGIATZIDAKIS, P.: Empirical Research in Mid-Air Interaction: A Systematic Review. Int. Journal of Human-Computer-Interaction, Vol. 19/2 (2019), S. 1-22.

[8] ALT, F., GEIGER, S., HÖHL, W.: ShapelineGuide Teaching Mid-Air gestures for large interactive Displays, Proc. $9^{\text {th }}$ ACM Int. Symp. On Pervasive Displays (PerDis'18), Munich 2018.

[9] LUNA, M., CARVALHO, Th., ALPHONSUS F., SOARES, A., NASCIMENTO, H., COSTA, R.: Wrist Player: a smartwatch gesture controller for smart TVs. IEEE Annual Software and Applications Conf., Turin, 2017.

[10] BOBETH, J., SCHRAMMEL, J., DEUTSCH, S., KLEIN, M., DROBICS, M., HOCHLEITNER, C., TSCHELIGI, M.: Tablet, gestures, remote control? Influence of age on performance and user experience with iTV applications. In: Proceedings of 2014 ACM International Conference on Interactive Experiences for TV and Online Video, 2014, S. 139-146.

[11] RUSER, H., KOSTERSKI, Sz., KARGEL, Ch.: Gesture-based universal optical remote control: Concept, reconstruction principle and recognition results. In: Tagungsband IEEE Int. Instrumentation and Measurement Technology Conf. (I ${ }^{2}$ MTC), Pisa, 2015.

[12] GOLUB, M: Laser beam splitting by diffractive optics, Opt. Photonics News 15(2), 2004, S. 36-41.

[13] VANDERHOUTEN, R., HERMERSCHMIDT, A., FIEBELKORN, R.: Design and quality metrics of point patterns for coded structured light illuminations with diffractive optical elements in optical 3D sensors, Proc. SPIE 10335, 2017.

[14] VORWERG, S., EICHER, C., RUSER, H., PIELA, F., OBĖE, F., KALTENBACH, A:, MECHOLD, L.: Requirements for Gesture-Controlled Remote Operation to Facilitate Human-Technology Interaction in the Living Environment of Elderly People. In: ZHOU, J., SALVENDY, G. (Hrsg.): 21st Int. Conf. on HumanComputer Interaction, LNSC 11592 (2019), S. 551569.

[15] ASADI-AGHBOLAGHI, M. et al., A survey on deep learning based approaches for action and gesture recognition in image sequences, 12the IEEE Int. Conf. on Automatic Face \& Gesture Recognition, Washington, 2017.

\section{Danksagung}

Die Arbeit wird vom BMBF im Rahmen der KMUInnovativ-Initiative „Mensch-Technik-Interaktion“ gefördert (Förderkennzeichen 16SV8009). Der Autor dankt C. Eicher und S. Vorwerg, Forschungsgruppe Geriatrie der Charité - Universitätsklinik Berlin, für die Planung und Begleitung der Gestenerfassung, A. Kaltenbach, S. Tischler und L. Mechold, Laser Components $\mathrm{GmbH}$, Olching, und F. Obée, F. Piela und dem Team von August\&Piela Konstruktiv GbR, Berlin, für ihre wertvolle Unterstützung sowie M. Horn, Ch. Kargel und Sz. Kosterski für ihre grundlegenden Beiträge. 